\title{
Polyvinylchloride-Single-Walled Carbon Nanotube Composites: Thermal and Spectroscopic Properties
}

\section{Mircea Chipara, ${ }^{1}$ Jessica Cruz, ${ }^{2}$ Edgar R. Vega, ${ }^{3}$ Jorge Alarcon, ${ }^{1}$ Thomas Mion, ${ }^{1}$ Dorina Magdalena Chipara, ${ }^{1}$ Elamin Ibrahim, ${ }^{2}$ Steven C. Tidrow, ${ }^{1}$ and David Hui ${ }^{4}$}

${ }^{1}$ Department of Physics and Geology, The University of Texas Pan-American, Edinburg, TX 78539, USA

${ }^{2}$ Department of Chemistry, The University of Texas Pan-American, Edinburg, TX 78539, USA

${ }^{3}$ Department of Mechanical Engineering, The University of Texas Pan-American, Edinburg, TX 78541, USA

${ }^{4}$ Department of Mechanical Engineering, University of New Orleans, LA 70148, USA

Correspondence should be addressed to Dorina Magdalena Chipara, dchipara@utpa.edu

Received 17 December 2011; Accepted 14 February 2012

Academic Editor: Sevan P. Davtyan

Copyright ( $) 2012$ Mircea Chipara et al. This is an open access article distributed under the Creative Commons Attribution License, which permits unrestricted use, distribution, and reproduction in any medium, provided the original work is properly cited.

\begin{abstract}
Nanocomposites of single-walled carbon nanotubes dispersed within polyvinylchloride have been obtained by using the solution path. High-power sonication was utilized to achieve a good dispersion of carbon nanotubes. Thermogravimetric analysis revealed that during the synthesis, processing, or thermal analysis of these nanocomposites the released chlorine is functionalizing the single-walled carbon nanotubes. The loading of polyvinylchloride by single-walled carbon nanotubes increases the glass transition temperature of the polymeric matrix, demonstrating the interactions between macromolecular chains and filler. Wide Angle XRay Scattering data suggested a drop of the crystallite size and of the degree of crystallinity as the concentration of single-walled carbon nanotubes is increased. The in situ chlorination and amorphization of nanotube during the synthesis (sonication step) is confirmed by Raman spectroscopy.
\end{abstract}

\section{Introduction}

Single-walled carbon nanotubes (SWNTs) are amazing onedimensional systems, characterized by semiconducting to conducting electrical conductivity $[1,2]$, excellent thermal conductivities [3], extremely large aspect ratio [3], impressing mechanical features (such as a Young modulus of the order of $1 \mathrm{TPa}$ ) $[4,5]$, and high thermal stability (mainly in inert atmosphere such as nitrogen) [6]. The radial breathing mode observed by Raman spectroscopy in single-walled carbon nanotubes (SWNTs) allows for a direct measurement of the diameter of SWNTs and of the effect of macromolecular chains on their diameter [7]. Typically, Raman lines are used to characterize carbon nanotubes and to monitor the interactions between nanotubes and polymeric matrices. Despite their restrictive cost, SWNTs are the filler of choice for many polymer-based nanocomposites [8, 9], due to their large aspect ratio, which determines a very low percolation threshold for the electrical conductivity and a larger enhancement of structural properties.

The addition of SWNTs to polymeric matrices results typically in an improvement of mechanical strength (in most cases associated with a drop in the value of the elongation at break) [8-10]. As carbon nanotubes and graphene are either semiconducting or conducting, the addition of such carbon nanostructures to insulating polymeric matrices increases the electrical conductivity (up to the point where electrostatic, electromagnetic shielding, or even electrical conduction is ignited in the polymer-based nanocomposites) [1114].

Typically, fillers with a larger aspect ratio are associated with a lower percolation threshold for the electrical conductivity (usually occurring at a concentration of about 1\% SWNTs in polymeric matrices) and with a better enhancement of the mechanical and structural features of the polymeric matrix than spherical fillers. Percolation theory 
describes accurately the effect of the concentration, aspect ratio, and electrical conductivity of the filler on the resulting electrical features of the polymer-based nanocomposite. The dielectric constant of polymer-based nanocomposites is typically enhanced by the addition of carbonaceous nanostructures, with a maximum around the percolation threshold for the electrical conductivity [15-17].

Carbon nanotubes have also an excellent thermal conductivity and good thermal stability, while most polymers exhibit a rather poor thermal conductivity and degrade under the effect of temperature. The presence of oxygen accelerates the thermal degradation of polymers. The loading of polymeric matrices with carbon nanotubes is usually increasing the thermal conductivity of the matrix. The addition of nanometer-sized fillers to polymeric matrices results typically in the enhancement of the thermal stability of the polymeric matrix [18-20]. Such improvements have been reported for poly(3-hexylthiophene)-single-walled carbon nanotubes nanotube composites [18], isotactic polypropylene-vapor grown carbon nanofibers [19], isotactic polypropylene-multiwalled carbon nanotubes [21], polyethylene-multiwalled carbon nanotubes [20], low-density polyethylene-nanoclays [21], and polystyrene-dimethyl decylimidazolium montmorillonite [21]. The above-mentioned modifications reflect the formation of an interface between the nanofiller and the polymeric matrix. Due to the huge area of the nanofillers, the weight of the polymer trapped within the interface can become comparable and even larger than the weight of macromolecular chains that are not in interaction with the nanometer-sized filler. Hence, the physical properties of the polymer nanocomposites start to become dominated by the features of the polymer-filler interface. This explains qualitatively the above-mentioned changes.

In the case of polyethyleneterephtalate filled singlewalled carbon nanotube composites, no enhancement of the thermal stability was noticed, while in the case of polyethyleneterephtalate filled with multiwalled carbon nanotubes a weak improvement was reported [22]. While single-walled carbon nanotubes have-at the same loading - a significantly larger total area than multiwalled carbon nanotubes, this anomalous behavior was tentatively assigned to the functionalization of multiwalled carbon nanotubes. Modifications of the thermal degradation process's path due to the nanofiller have been reported (see e.g., the nanocomposite ethylene vinyl acetate-modified nanoclay) [21]. Preliminary studies on multiwalled carbon nanotubes grafted on polyvinyl chloride (PVC) revealed an enhancement of the thermal stability of the matrix upon crosslinking [23]. Nanocomposites of poly(2,5-benzoxazole)-multiwalled carbon nanotubes obtained by in situ polycondensation showed a weak increase of the thermal stability of the polymeric matrix [15].

PVC is a polymer used frequently in applications that require structural features (mechanical strength) or electrical insulating capabilities [24]. The use of SWNTs degrades the electrical insulating features of PVC, and hence the most important application for such nanocomposites aims at structural capabilities. An important limitation in the use of PVC derives from its weak resistance to fire and thermal degradation in general $[24,25]$. Under the effect of temperature, PVC exhibits a fast degradation associated with the release of chlorine and hydrogen as the most important volatile products. These gases can interact resulting in a local concentration of $\mathrm{HCl}$. The dehydrochlorination reaction is initiated by chain end groups (eventually containing initiator residues or unsaturated groups), branch points involving tertiary chlorine atoms, random unsaturation (such as allylic chlorine atoms), and head-to-head units. Oxidation structures are playing an important role if the thermal degradation occurs in oxygen-containing atmosphere.

In nitrogen atmosphere, SWNTs have an excellent thermal stability, with a low weight loss below $700^{\circ} \mathrm{C}$. Nevertheless, SWNTs are prone to the chlorine functionalization [26], achieved through the local thermal decomposition of the polymeric matrix. The thermal stability of SWNTs is significantly lower in oxygen containing atmosphere.

\section{Experimental Methods}

2.1. Materials. $\mathrm{PVC}$ with an average molecular mass, $\mathrm{Mn}=$ 47,000, was purchased from Sigma Aldrich. SWNTs, with a purity of $90 \%$, outer diameters $1-2 \mathrm{~nm}$, and lengths ranging between 5 and 30 microns, were purchased from CheapTubes. Research grade tetrahydrofuran (THF), used as solvent for PVC, was purchased from Sigma Aldrich and utilized as received.

2.2. Methods. Homogeneous solutions of PVC have been obtained by dissolving the polymer in THF followed by stirring at 500 rotations per minute for 8 hours, at room temperature. Various amounts of SWNTs were added to the solution of PVC and stirred for about 2 hours. After that, the liquid mixture PVC, THF, and SWNTs was sonicated for one hour by using a Hielscher high power sonicator $(1 \mathrm{KW})$. The as obtained fluid was cast on microscope slides, and the solvent was removed by heating at $100^{\circ} \mathrm{C}$ for about 4 hours.

Thermogravimetric analysis (TGA) data were obtained using a TA Instrument TGA 500 instrument, Differential Scanning Calorimetry (DSC) investigations were performed with a TA Instrument DSC Q500, Raman investigations were done by using a Bruker Sentera microRaman operating at $785 \mathrm{~nm}$, and Wide Angle X-Rays Scattering (WAXS) analysis was performed by a Bruker 8 Discovery spectrometer.

\section{Results and Discussions}

Nanocomposites of polyvinylchloride-single-walled carbon nanotubes (PVC-SWNTs) containing various weight fractions of SWNTs ranging from $0 \%$ to $20 \% \mathrm{wt}$. were obtained. The total removal of the solvent was confirmed using TGA, which showed no changes in the mass of PVC-SWNTs up to $150^{\circ} \mathrm{C}$ (within an accuracy of $0.1 \%$ ). The boiling temperature of THF is about $66^{\circ} \mathrm{C}$.

3.1. Thermal Investigations. TGA investigations, in nitrogen, have been performed at a heating rate of $10^{\circ} \mathrm{C}$ from room temperature to about $650^{\circ} \mathrm{C}$. The pristine polymer (PVC) shows a typical two steps thermal degradation (see 


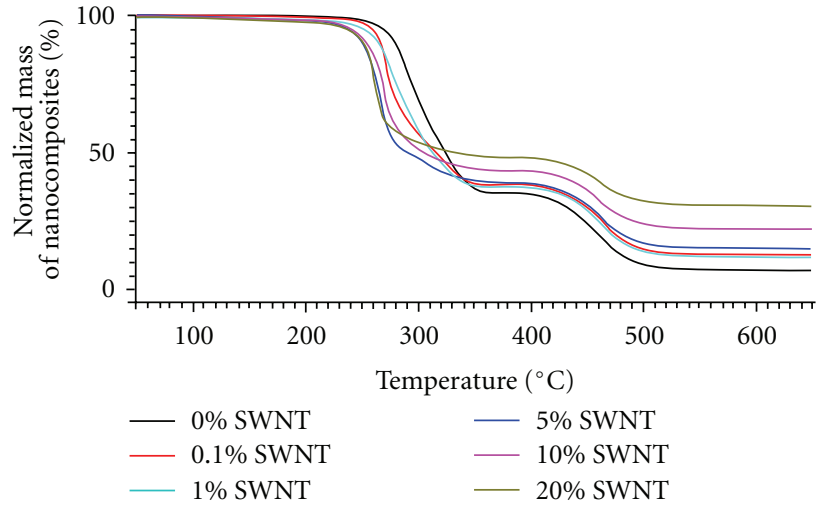

(a)

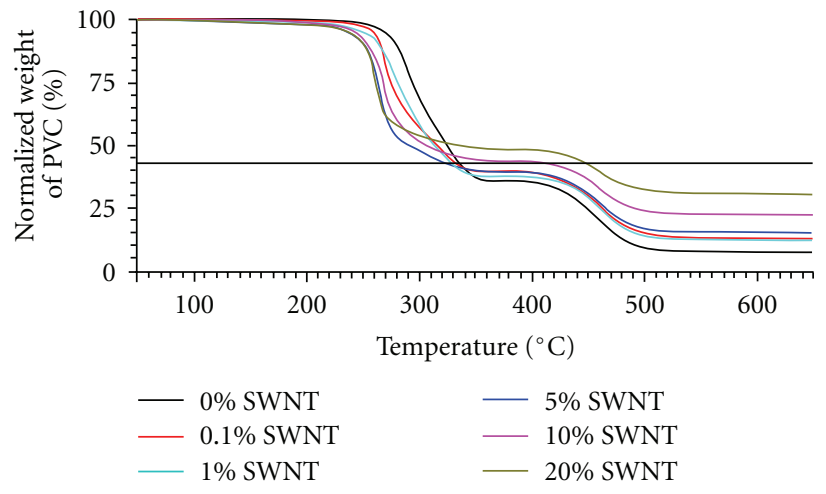

(b)

Figure 1: (a) The evolution of the total mass (PVC + SWNTs) of the nanocomposite versus temperature. (b) The evolution of the polymeric part (PVC) of the nanocomposite versus temperature.

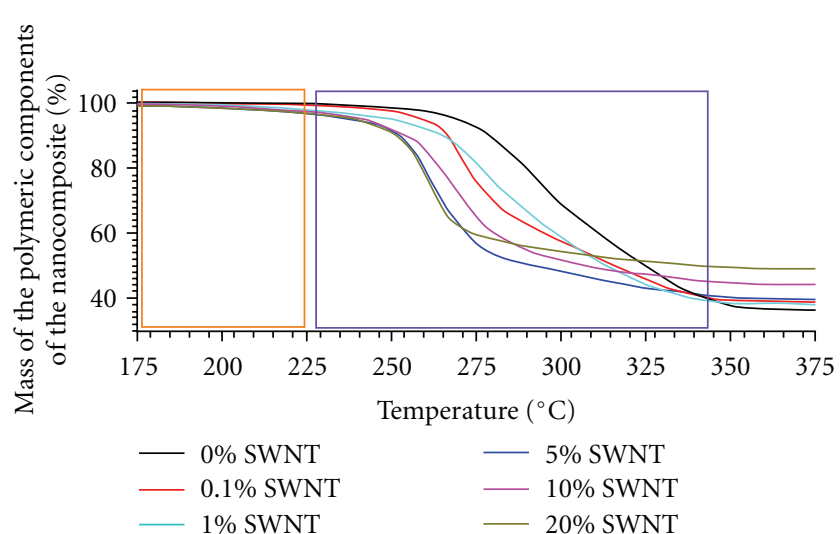

(a)

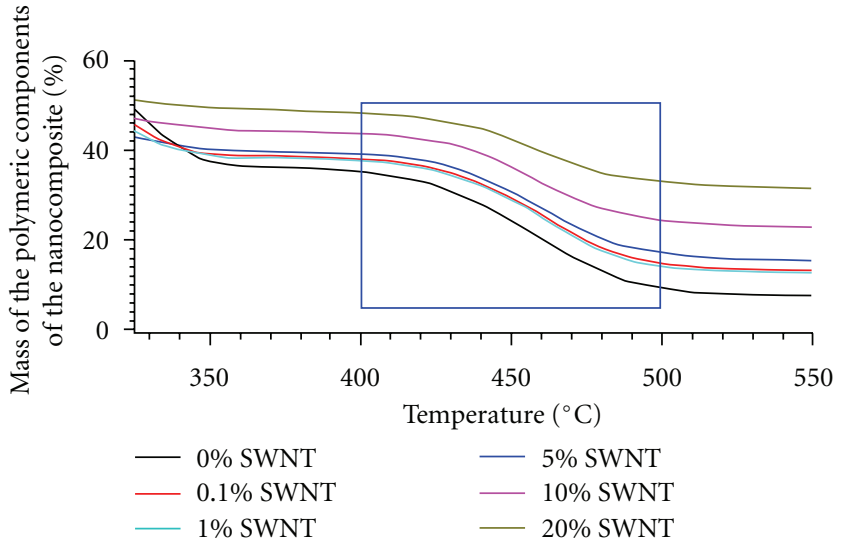

(b)

FIGURE 2: Details of the thermal degradation of PVC-SWNTs in nitrogen atmosphere (time dependence of the mass of the polymeric component). (a) Low temperature degradation. (b) High temperature degradation.

Figure 1(a)), one dominated by the dehydrochlorination of the sample and the last representing the final thermal degradation of the polymer $[24,25,27]$. The thermal degradation of PVC in nitrogen lefts a certain carbon residue. The dependence of the weight of PVC-SWNTs nanocomposites on temperature is shown in Figure 1(a). It is noticed that the degradation has a rather complex dependence on the concentration of SWNTs and occurs in two steps.

For a better understanding of TGA data, it is necessary to observe that SWNTs are typically not degraded in nitrogen up to about $600^{\circ} \mathrm{C}$ [28]. The degradation of SWNTs in an inert atmosphere in the range 400 to $600^{\circ} \mathrm{C}$ is rather slow and weak [28]. As this is the case for our filler, we can represent the dependence of the mass of the polymeric component (i.e., solely the mass of PVC) of PVC-SWNTs on the degradation temperature (see Figure 1(b)). If the contribution of the effects due to the polymer-filler interface is negligible and the SWNTs can be assumed to be inert during the thermal degradation in nitrogen of these nanocomposites, then all thermograms shown in Figure 1(b) should overlap. This is obviously not the case, suggesting important contributions due to the loading of the polymer with SWNTS. Due to the large atomic mass of chlorine, a full dehydrochlorination of PVC would result in the volatilization of about $65 \%$ of the pristine polymer (see the line in Figure 1(b)). The fact that the first thermal degradation process leaves more residues suggests that some chlorine is transferred to nanotubes.

The first step of the thermal degradation of PVC-SWNTs is shown in Figure 2(a). It is noticed that the degradation starts at about $175^{\circ} \mathrm{C}$ and becomes stronger in the temperature range from 225 to $350^{\circ} \mathrm{C}$. Between $350^{\circ} \mathrm{C}$ and $425^{\circ} \mathrm{C}$, there is a weak decrease of the mass of these samples as the temperature is increased (below 5\%). The temperature range (after the completion of the first degradation step) over which the mass of the polymer component of the composite is almost temperature independent narrows as the concentration of SWNTs is increased (see Figures 2(a) and 2(b)). As the temperature is further increased, a secondary degradation process is ignited (see Figure 2(b)). The degradation is essentially occurring in the temperature range from $425^{\circ} \mathrm{C}$ 
to $500^{\circ} \mathrm{C}$. Further increase of the temperature of the sample above $500^{\circ} \mathrm{C}$ (up to $650^{\circ} \mathrm{C}$ ) does not result in a significant drop of the mass of the sample. For the pristine PVC, the amount of residual char at $650^{\circ} \mathrm{C}$ represents about $7 \%$ of the mass of the initial sample. In the case of PVC-SWNTs composites, the amount of char is larger as the degradation of single-walled carbon nanotubes in inert atmosphere (nitrogen) is typically ignited above $500^{\circ} \mathrm{C}$ [28].

The residual mass of the polymeric component PVCSWNTs containing $20 \%$ SWNTs after the thermal degradation in nitrogen up to $650^{\circ} \mathrm{C}$ is about $30 \%$, significantly larger than the expected value. This supports the idea that an important fraction of the chlorine released during the thermal degradation of the polymer was captured by nanotubes. A complex adsorption desorption of chlorine compounds on carbon nanotubes has been reported elsewhere [29]. In conclusion, some of the chlorine released during the first degradation step functionalized the SWNTs. From Figure 2(a), it is noticed that for the pristine sample, the residual mass at the end of the first degradation [27] step (at $275^{\circ} \mathrm{C}$ ) is of about $35 \%$, suggesting an almost complete dehydrochlorination, while the sample containing $20 \%$ SWNTs exhibits a residual polymeric component of about $50 \%$. This reflects the complex thermal degradation of PVC-SWNTs samples, where probably SWNTs are catalyzing the dehydrochlorination reaction facilitating the transfer of chlorine from the macromolecular chain to the nanotube and destabilizing the macromolecular chain. We are speculating the fact that the degraded PVC-SWNTs contain a higher concentration of chlorine and eventually hydrogen plus eventually short polymeric chains. The chlorination of carbon nanotube was reported elsewhere [26]. A complex effect of nanoclay fillers on the thermal degradation of PVC was recently reported [30].

Differential Scanning Calorimetry data in the temperature range from $0^{\circ} \mathrm{C}$ to $150^{\circ} \mathrm{C}$ revealed solely the glass transition temperature. The upper temperature was restricted to about $150^{\circ} \mathrm{C}$, in order to avoid the thermal degradation of the sample. However, due to the thermal degradation and instrumental inertia/hysteresis, solely the temperature range fom 25 to $125^{\circ} \mathrm{C}$ contains reliable experimental data. No melting or crystallization peaks were noticed in this temperature range. Due to the high molecular mass of the polymeric matrix, it is expected that these transition points are over $150^{\circ} \mathrm{C}$. The glass transition temperatures for the third cycle (second heating) are almost identical with the fifth thermal cycle (third heating) for all samples. However, Figure 3 shows only the data for the pristine sample and two nanocomposites containing 5 and 20\% (wt.) SWNTs. These results confirm that the potential contributions of thermal stresses were completely removed and the experimental data are accurate. The position of the glass transition was determined by the slope method [31]. The estimated glass transition temperature $\left(T_{G}\right)$ of the pristine polymer was about $88.5^{\circ} \mathrm{C}$, in agreement with experimental data reported elsewhere [19]. The inset of Figure 3 depicts the effect of SWNTs on the glass transition of PVC. An increase of $T_{G}$ by about $10^{\circ} \mathrm{C}$ was noticed while the concentration of SWNTs is increased from $0 \%$ to $20 \%$ (wt.). Similar results were reported for PVC

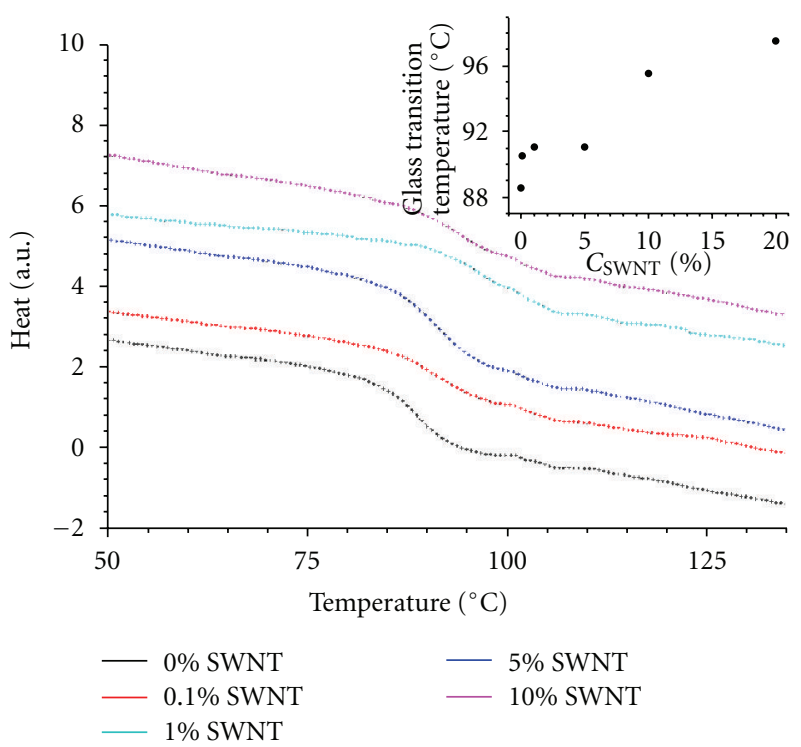

FIgURE 3: DSC data on PVC-SWNT nanocomposites showing the effect of the loading with SWNTs, at a heating rate of $20^{\circ} \mathrm{C} /$ minute. The inset shows the dependence of the glass transition on the concentration of SWNTs.

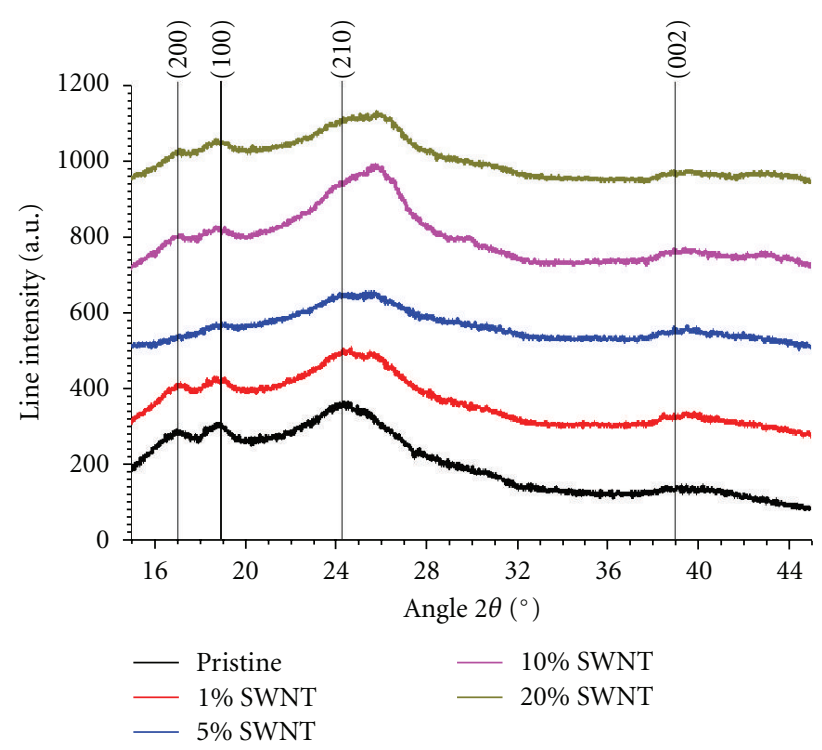

Figure 4: WAXS spectra of pristine PVC and PVC-SWNTs composites.

loaded by multiwalled carbon nanotubes [32]. This suggests that nanotubes are sharing some free volumes with the macromolecular chains, generating an interface region within which the segmental motions are hindered.

3.2. Spectroscopic Investigations. PVC is a semicrystalline polymer with a rather poor WAXS spectrum. As seen in Figure 4, the nanocomposites exhibit the most intense peaks: 200 located at about $17^{\circ}, 110$ located at about $19^{\circ}$, and 210 located at about $24^{\circ}[33,34]$. A broad and weak peak due to SWNTs, located at about $26^{\circ}$, is convoluted with the 210 


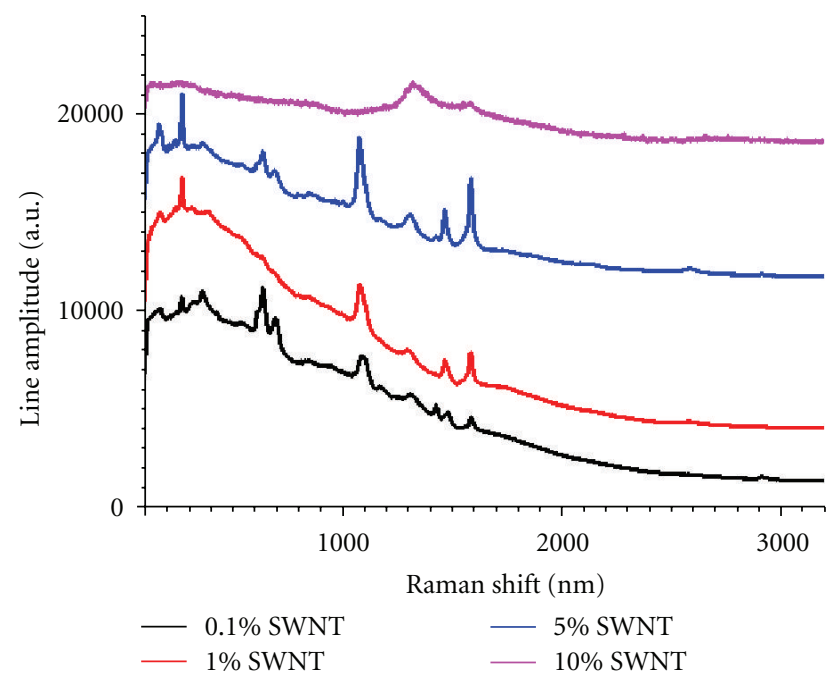

Figure 5: Raman spectra of some PVC-SWNTs.

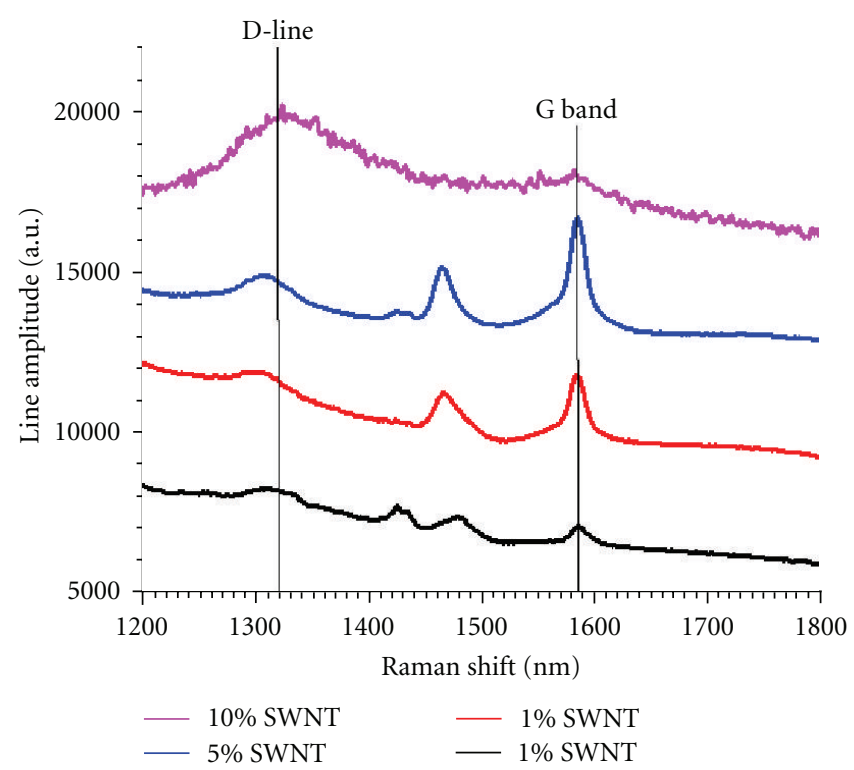

FIGURE 6: Raman spectra of some PVC-SWNT nanocomposites (detail showing the D and G lines of SWNTs).

reflection. Figure 4 suggests that the loading with SWNTs broadens the lines, decreasing the dimensions of polymeric crystallites.

Raman Spectra of some PVC-SWNTs composites are shown in Figure 5. It is observed that the Raman spectrum of pristine polymer is sensitive to the addition of SWNTs. Even more, the lines due to SWNTs are significantly distorted at high SWNTs content (see Figure 6), due to the chlorination (see in Figure 6 the changes of the G band-a band that is sensitive to the electric conductivity of SWNTs [7]). The ratio between $\mathrm{D}$ and $\mathrm{G}$ bands is a qualitative measure of the degree of disorder of the carbon nanotubes. An important increase of this ratio is noticed for the samples containing $10 \%$ wt. SWNTs (see Figure 6). This suggests an important chlorination of SWNT even during the preparation of the nanocomposite. The high power sonication can generate small bubbles at the interface polymer-solution-SWNTs, and the cavitation effect may result in large local temperature, capable of generating $\mathrm{Cl}$ atoms near the surface of the nanotube.

\section{Conclusions}

PVC-SWNTs nanocomposites have been investigated. Their thermal degradation in nitrogen is complex due to the competition between the dehydrochlorination of macromolecular chains, the chlorination of SWNTs, and the complex sorption-desorption phenomena involving chlorinated compounds. In contrast with the results reported for PVC chains grafted onto multiwalled carbon nanotubes [23], we did not observe any enhancement in the thermal stability of the polymeric matrix upon the filling with SWNTs.

Raman data demonstrates that an important chlorination and amorphization of SWNTs occurs during the sonication step. The increase of the $T_{G}$ as the concentration of SWNTs is increased reveals the effect of crosslinking reactions as well as the eventual competition between segments and nearest nanotubes for the same free volume. WAXS data revealed a decrease of PVC crystallites size as the concentration of SWNTs is increased.

The existing data suggests that such nanocomposites can be also used either as antistatic materials or conducting polymer-based nanocomposites. Electrical studies to determine the percolation threshold are in progress. Further research is scheduled to assess the effect of the nanofiller on the mechanical properties and to determine the potential performances of PVC-SWNTs as structural materials. Nevertheless, as the loading of PVC with SWNTs does not enhance the thermal stability of the polymeric matrix, all applications of PVC should be restricted to environments that do not exceed $150^{\circ} \mathrm{C}$.

\section{Acknowledgments}

This paper has been supported by the Faculty Research Council Grant (University of Texas Pan American, 135PHYS04), National Science Foundation Partnership for Research and Education in Materials awarded to UTPA University of Minnesota, by the Division of Materials Research Grant no. 0934157 NSF, by the U.S. Army Research Laboratory and the U.S. Army Research Office under contract/Grant no. W911NF-08-1-0353, and Welch Foundation Grant no. BG-0017, awarded to the Chemistry Department of UTPA.

\section{References}

[1] J. Bernholc, D. Brenner, M. Buongiorno Nardelli, V. Meunier, and C. Roland, "Mechanical and electrical properties of nanotubes," Annual Review of Materials Science, vol. 32, pp. 347375, 2002.

[2] M. Ouyang, J.-L. Huang, and C. M. Lieber, "Scanning tunneling microscopy studies of the one-dimensional electronic properties of single-walled carbon nanotubes," Annual Review of Physical Chemistry, vol. 53, pp. 201-220, 2002. 
[3] M. S. Dresselhaus, G. Dresselhaus, and A. Jorio, "Unusual properties and structure of carbon nanotubes," Annual Review of Materials Research, vol. 34, pp. 247-278, 2004.

[4] S. Kirtania and D. Chakraborty, "Finite element based characterization of carbon nanotubes," Journal of Reinforced Plastics and Composites, vol. 26, no. 15, pp. 1557-1570, 2007.

[5] M. Terrones, "Science and technology of the twenty-first century: synthesis, properties, and applications of carbon nanotubes," Annual Review of Materials Research, vol. 33, no. 1, pp. 419-501, 2003.

[6] H. M. Duong, E. Einarsson, J. Okawa, R. Xiang, and S. Maruyama, "Thermal degradation of single-walled carbon nanotubes," Japanese Journal of Applied Physics, vol. 47, no. 4, pp. 1994-1999, 2008.

[7] D. M. Chipara, A. C. Chipara, and M. Chipara, "Raman spectroscopy of carbonaceous materials: a concise review," Spectroscopy, vol. 26, no. 10, pp. 2-7, 2011.

[8] F. Hussain, "Review article: polymer-matrix nanocomposites, processing, manufacturing, and application: an overview," Journal of Composite Materials, vol. 40, no. 17, pp. 1511-1575, 2006.

[9] K. Suzuki and S. Nomura, "On elastic properties of singlewalled carbon nanotubes as composite reinforcing fillers," Journal of Composite Materials, vol. 41, no. 9, pp. 1123-1135, 2007.

[10] C.-J. Chou, A. E. Read, and C. P. Bosnyak, "Polymer nanocomposite," Polymer, no. June, pp. 11-13, 2002.

[11] J. Li and J. K. Kim, "Percolation threshold of conducting polymer composites containing 3D randomly distributed graphite nanoplatelets," Composites Science and Technology, vol. 67, no. 10, pp. 2114-2120, 2007.

[12] N. Li, Y. Huang, F. Du et al., "Electromagnetic interference (EMI) shielding of single-walled carbon nanotube epoxy composites," Nano Letters, vol. 6, no. 6, pp. 1141-1145, 2006.

[13] F. Du, J. E. Fischer, and K. I. Winey, "Effect of nanotube alignment on percolation conductivity in carbon nanotube/polymer composites," Physical Review B, vol. 72, no. 12, pp. 1-4, 2005.

[14] T. Ramanathan, A. A. Abdala, S. Stankovich et al., "Functionalized graphene sheets for polymer nanocomposites," Nature Nanotechnology, vol. 3, no. 6, pp. 327-331, 2008.

[15] Z. Xie, Q. Zhuang, Q. Wang, X. Liu, Y. Chen, and Z. Han, "Insitu synthesis and characterization of poly(2,5-benzoxazole)/ multiwalled carbon nanotubes composites," Polymer, vol. 52, no. 23 , pp. 5271-5276, 2011.

[16] J. Macutkevic, D. Seliuta, G. Valusis et al., "Effect of thermal treatment conditions on the properties of onion-like carbon based polymer composite," Composites Science and Technology, vol. 70, no. 16, pp. 2298-2303, 2010.

[17] J. Macutkevic, P. Kuzhir, D. Seliuta et al., "Dielectric properties of a novel high absorbing onion-like-carbon based polymer composite," Diamond and Related Materials, vol. 19, no. 1, pp. 91-99, 2010.

[18] A. R. Adhikari, M. Huang, H. Bakhru, M. Chipara, C. Y. Ryu, and P. M. Ajayan, "Thermal property of regioregular poly(3hexylthiophene)/nanotube composites using modified singlewalled carbon nanotubes via ion irradiation," Nanotechnology, vol. 17, no. 24, pp. 5947-5953, 2006.

[19] M. Chipara, K. Lozano, and A. Hernandez, "TGA analysis of polypropylene-carbon nanofibers composites," Polymer Degradation and Stability, vol. 93, no. 4, pp. 871-876, 2008.

[20] A. R. Adhikari, M. Chipara, and K. Lozano, "Processing effects on the thermo-physical properties of carbon nanotube polyethylene composite," Materials Science and Engineering A, vol. 526, no. 1-2, pp. 123-127, 2009.
[21] K. Chrissafis and D. Bikiaris, "Can nanoparticles really enhance thermal stability of polymers? Part I: an overview on thermal decomposition of addition polymers," Thermochimica Acta, vol. 523, no. 1-2, pp. 1-24, 2011.

[22] D. Bikiaris, "Can nanoparticles really enhance thermal stability of polymers? Part II: an overview on thermal decomposition of polycondensation polymers," Thermochimica Acta, vol. 523, no. 1-2, pp. 25-45, 2011.

[23] X. L. Wu, "Poly(vinyl chloride)-grafted multi-walled carbon nanotubes via Friedel-Crafts alkylation," Express Polymer Letters, vol. 4, no. 11, pp. 723-728, 2010.

[24] Y. Soudais, L. Moga, J. Blazek, and F. Lemort, "Coupled DTATGA-FT-IR investigation of pyrolytic decomposition of EVA, PVC and cellulose," Journal of Analytical and Applied Pyrolysis, vol. 78, no. 1, pp. 46-57, 2007.

[25] M. J. P. Slapak, J. M. N. van Kasteren, and A. A. H. Drinkenburg, "Determination of the pyrolytic degradation kinetics of virgin-PVC and PVC-waste by analytical and computational methods," Computational and Theoretical Polymer Science, vol. 10, no. 6, pp. 481-489, 2000.

[26] M. Chipara, R. Wilkins, E. V. Barrera, and M. D. Chipara, "ESR investigations on polyethylene-fluorine functionalized single wall carbon nanotubes composites," Composite Interfaces, vol. 17, no. 5-7, pp. 625-632, 2010.

[27] P. S. K. Pielichowski, I. Hamerton, J. Pielichowski, and P. Stanczyk, "A study of the thermal properties of blends of poly(vinyl chloride) with novel epoxypropanecarbazole-based dyes by TGA/FTIR," European Polymer Journal, vol. 34, no. 56, pp. 653-657, 1998.

[28] M. Zhang, M. Yudasaka, A. Koshio, and S. Iijima, "Thermogravimetric analysis of single-wall carbon nanotubes ultrasonicated in monochlorobenzene," Chemical Physics Letters, vol. 364, no. 3-4, pp. 420-426, 2002.

[29] X. Ma, D. Anand, X. Zhang, and S. Talapatra, "Adsorption and desorption of chlorinated compounds from pristine and thermally treated multiwalled carbon nanotubes," Journal of Physical Chemistry C, vol. 115, no. 11, pp. 4552-4557, 2011.

[30] W. H. Awad, G. Beyer, D. Benderly et al., "Material properties of nanoclay PVC composites," Polymer, vol. 50, no. 8, pp. 1857-1867, 2009.

[31] E. Bureau, C. Cabot, S. Marais, and J. M. Saiter, "Study of the $\alpha$-relaxation of PVC, EVA and 50/50 EVA70/PVC blend," European Polymer Journal, vol. 41, no. 5, pp. 1152-1158, 2005.

[32] T. Sterzyński, J. Tomaszewska, K. Piszczek, and K. Skórczewska, "The influence of carbon nanotubes on the PVC glass transition temperature," Composites Science and Technology, vol. 70, no. 6, pp. 966-969, 2010.

[33] R. J. Hobson and A. H. Windle, "Crystallization and shape emulation in atactic poly(vinyl chloride) and polyacrylonitrile," Polymer, vol. 34, no. 17, pp. 3582-3596, 1993.

[34] Y. Liu and C. Zhang, "The influence of additives on crystallization of polyvinyl chloride," Journal Wuhan University of Technology, Materials Science Edition, vol. 22, no. 2, pp. 271275, 2007. 

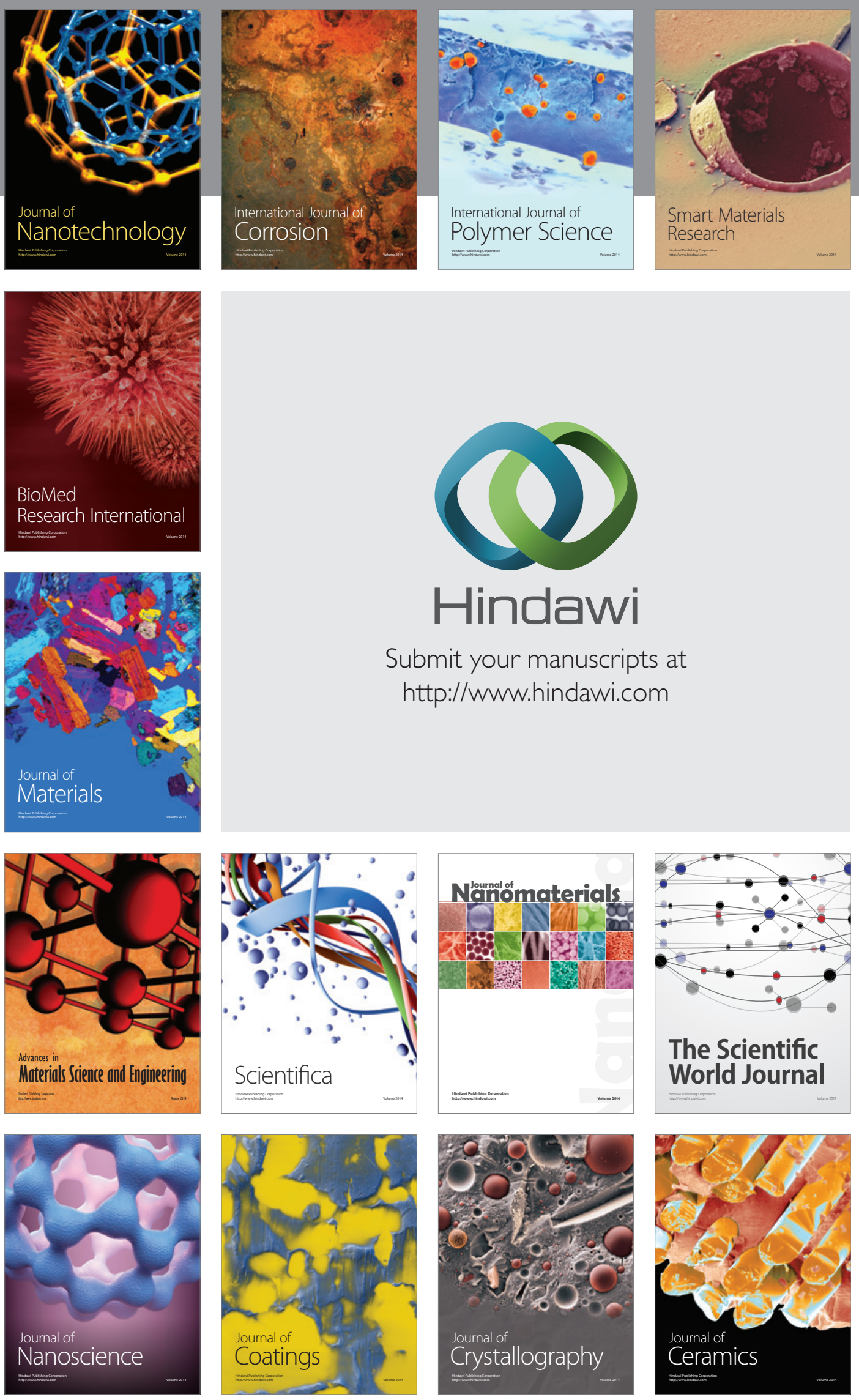

The Scientific World Journal

Submit your manuscripts at

http://www.hindawi.com

\section{World Journal}

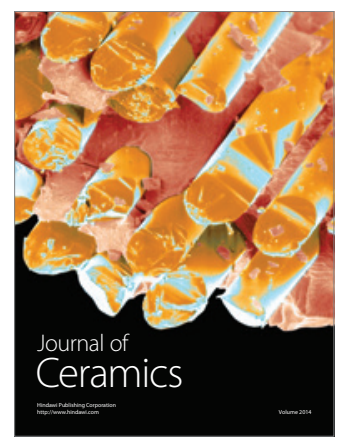

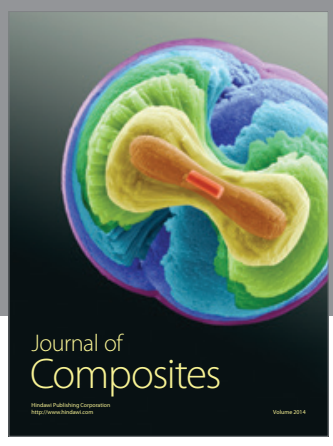
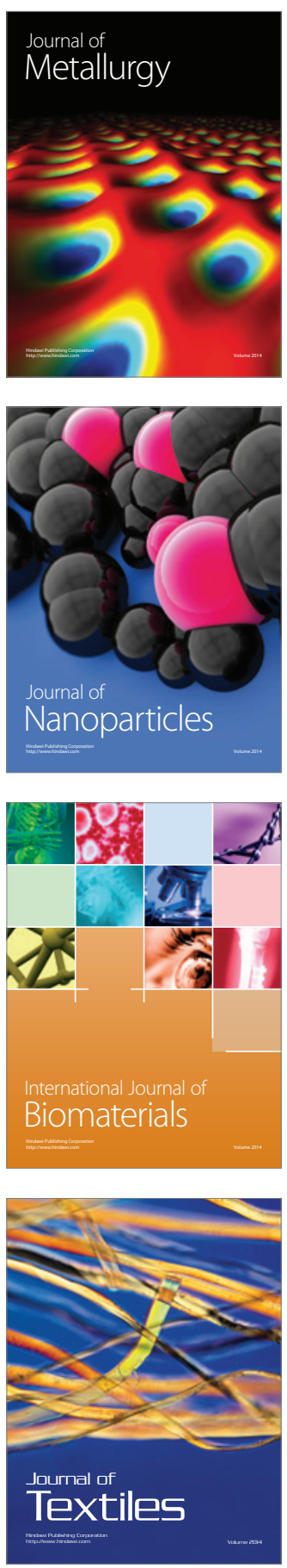